\title{
BMJ Open What is known about the health service use and follow-up of immediate family members bereaved by suicide? Scoping review protocol
}

\author{
Sindre Hoff Petersen (1) , Jorid Kalseth, Silje L Kaspersen
}

To cite: Petersen SH, Kalseth J, Kaspersen SL. What is known about the health service use and follow-up of immediate family members bereaved by suicide? Scoping review protocol. BMJ Open 2020;10:e041978. doi:10.1136/ bmjopen-2020-041978

- Prepublication history and additional materials for this paper are available online. To view these files, please visit the journal online (http://dx.doi. org/10.1136/bmjopen-2020041978).

Received 22 June 2020 Revised 12 November 2020 Accepted 20 November 2020
Check for updates

(C) Author(s) (or their employer(s)) 2020. Re-use permitted under CC BY-NC. No commercial re-use. See rights and permissions. Published by BMJ.

Department of Health Research, SINTEF, Trondheim, Norway

Correspondence to

Dr Silje L Kaspersen; silje.I.kaspersen@sintef.no

\section{ABSTRACT}

Introduction Suicide remains a major public health issue around the world. People bereaved by suicide are a vulnerable group who are at considerable risk of developing mental and physical health problems, such as complicated grief, post-traumatic stress disorder or cardiovascular disease. Many unanswered questions remain, in particular, in terms of their use of healthcare services. This protocol describes how we aim to systematically scope the existing literature on the professional follow-up and health service use by families bereaved by suicide. The scoping review will help to identify research gaps in the literature and aid in the planning and commission of future research. We will provide a summary of research findings.

Methods and analysis We will use the scoping review framework provided by the Joanna Briggs Institute. The Preferred Reporting Items for Systematic Reviews and Meta-Analyses Extension for Scoping Reviews will be used as a guide for reporting our results. We plan to conduct an extensive literature search using relevant health-related databases (MEDLINE, Embase, PsycINFO and CINAHL) and Web of Science. Two independent reviewers will screen the articles in a two-stage process: (1) titles and abstracts and (2) full-text documents.

Ethics and dissemination This scoping review will identify and consider only previously published research. Hence, no ethical approval is considered necessary. We will disseminate the results in a scientific journal and at conferences, as well as through user organisations for people bereaved by suicide and social media.

\section{INTRODUCTION}

Suicide remains a major public health issue throughout the world, and suicide rates have increased in several countries during the past years. ${ }^{1}$ Despite continued efforts in prevention and research, suicide accounts for close to 800000 deaths annually worldwide and is one of the main causes of death in young adults. ${ }^{2}$ With the COVID-19 pandemic potentially introducing a worldwide recession and increasing unemployment rates, concerns have now been raised internationally about potential increase in suicide rates. ${ }^{3}$

\section{Strengths and limitations of this study}

- This scoping review aims to scope the existing literature on the professional follow-up and health service use by families bereaved by suicide, an outcome which seems to be scarcely studied in the existing literature.

- The search strategy will include searching through five major electronic databases of the peer-reviewed literature, using search strings on keywords through an iterative process, as well as consultation with a health sciences librarian.

- We will follow the guidance of the Joanna Briggs Institute with regard to the scoping review framework, and the Preferred Reporting Items for Systematic Reviews and Meta-Analyses Extension for Scoping Reviews checklist and explanation when conducting this scoping review.

- Important limitations are that bereaved in the non-immediate family/friends/colleagues, the nonEnglish and grey literature (eg, book chapters, reports and national guidelines) will be excluded, which means we may miss potential important sources of evidence.

- Our review will include only studies from highincome countries, which is a limitation but at the same time increases the probability of comparable levels of healthcare services in the included literature.

Information is needed urgently to help to prevent suicide and to know how best to help and support people bereaved by suicide. In this protocol, we describe a forthcoming scoping review that will investigate the follow-up and professional support of families bereaved by suicide and their patient trajectories in the health services over both the short and long term.

For each suicide, it is estimated that at least six close family members are left behind. These family members are referred to as people bereaved by suicide. ${ }^{4-6}$ Some studies have even estimated that an average of 135 
people may be affected by each suicide, although how best to estimate this number is an ongoing debate. ${ }^{5}$ In 2014, Pitman et al estimated that 48-500 million people worldwide experience suicide bereavement every year. ${ }^{6}$ Until recently, only a small proportion of the general suicidology literature has focused on the outcomes, needs and characteristics of families bereaved by suicide. ${ }^{7}$ The few studies published have focused mainly on specific health outcomes and postvention (i.e. the support and follow-up of people bereaved by suicide) ${ }^{8}$

In addition to the general grief and reactions related to the loss of a loved one, people bereaved by suicide may be at risk of developing complicated grief reactions, as well as long-term mental and physical health problems. ${ }^{6910} \mathrm{In}$ particular, this population may struggle with feelings of shame and blame, social stigma, rejection and abandonment. ${ }^{6}$ Compared with the general population, people bereaved by suicide are at two to three times higher risk of suicidal behaviour. ${ }^{11}$ A Norwegian study found that $80 \%$ of suicide-bereaved parents reported that they were in need of long-term medical or psychological care. ${ }^{12}$ Bereaved children after parental suicide may also be at risk, as shown by their increased risk of depression, poor educational performance and suicide. ${ }^{6} 1314$ Dyregrov found that nearly $70 \%$ of suicide bereaved adolescents reported a need for professional psychological assistance. ${ }^{15}$ However, knowledge about the specific needs and patient pathways through health service use in both short and long terms of families bereaved by suicide remains scarce.

Several systematic and mapping reviews on suicide bereavement, with varying themes, have been reported. In 2018, Maple et al mapped the suicide bereavement and postvention literature and characterised 443 articles in terms of their methodological design, year of publication, age and population groups, and geographical spread. ${ }^{16}$ In 2019, Bartone et al systematically reviewed the literature on the effects of peer support for bereaved survivors, including counselling from peers, support groups and internet forums. ${ }^{17}$ Andriessen et al reviewed the evidence for the effectiveness of several interventions for people bereaved by suicide. ${ }^{18}$ However, few of the articles captured by the these reviews had healthcare use as the main outcome.

This article describes the protocol for a scoping review to examine the existing literature on the follow-up of families bereaved by suicide and their patient pathways, experiences and outcomes in the health services. The objectives of the scoping review were as follows: (1) to scope the extent and nature of the scientific literature on suicide bereavement and healthcare use and follow-up in the health services, (2) to synthesise the findings in the literature to give an overview of patient trajectories and the interventions/initiatives provided in the health services and their potential effect/effectiveness for families bereaved by suicide and (3) to identify research gaps to suggest further research that will be helpful for the education of health professionals and clinical practice.

\section{Study rationale}

This scoping review constitutes a part of an ongoing research project ('Treatpath') led by the Norwegian Institute of Public Health, in collaboration with Department of Health Research, SINTEF, and Oslo University Hospital. The overall aim of Treatpath is to investigate the healthcare use and patient trajectories in both those who have committed suicide and their immediate family members in order to use this knowledge to contribute to the improved prevention of suicide and follow-up of families bereaved by suicide. To provide an overview of the existing international research, summarise findings and identify knowledge gaps, we will start the project by conducting a scoping review. The review will provide the basis for further development of research questions and practice recommendations within the project. A preliminary literature search while we were formulating this scoping review protocol has shown that there is limited knowledge on the role health services and professionals play in the follow-up and support of people bereaved by suicide. Furthermore, health services are constantly evolving, for example, through technological development and digitalisation of certain services, which necessitate frequent updates of the review literature. A PubMed search on the terms "Suicide" AND "Bereave*" show that the number of publications on suicide bereavement has increased in the past few years, but no recent reviews have been published on the specific topic health service use.

\section{METHODS AND ANALYSIS}

\section{Patient and public involvement}

The National Association of Suicide Bereaved in Norway (LEVE) has been, and will be, systematically involved in all phases of the Treatpath project, including the application process for project funding and recruitment of study participants. LEVE has a representative in the project's advisory board, which meets one to two times a year throughout the study period 2019-2022. LEVE highly welcomes more research in the field of suicide bereavement. LEVE will be asked to disseminate the results from the forthcoming scoping review to their members by email, meetings/seminars and social media.

\section{Stages in the scoping review}

The research literature on people bereaved by suicide appears to be diverse despite the limited focus on bereaved in the general suicidology literature. Hence, we consider that the framework of a scoping review is the most appropriate approach for reviewing this topic. We will use the scoping review framework provided by the Joanna Briggs Institute (JBI) ${ }^{19}$ which in turn is based on earlier efforts by Arksey and $\mathrm{O}^{\prime}$ Malley ${ }^{20}$ and Levac and colleagues. ${ }^{21}$ The JBI approach to conducting and reporting scoping reviews described here is congruent with the Preferred Reporting Items for Systematic Reviews and MetaAnalyses Extension for Scoping Reviews (PRISMA-ScR) checklist. ${ }^{1922}$ This will be used as a guide for reporting 
the results. ${ }^{23}$ The scoping review framework will comprise the following six stages: (1) identifying the research question; (2) identifying relevant studies; (3) study selection; (4) charting the data; (5) collating, summarising and reporting the results; and (6) consulting (optional). The following sections describe these steps in further detail. The literature search will be conducted in June 2020, and the scoping review article will presumably be submitted by spring 2021 .

\section{Stage 1: identifying the research question}

In accordance with the objectives of the Treatpath research project, we identified a need for summarised knowledge on use of/follow-up in the health services of families bereaved by suicide. Aims and study rationale are given in the introduction of this protocol. The main outcomes for which data will be sought are health service use and follow-up of immediate family members bereaved by suicide.

In this review, bereaved will be limited to immediate family. This limitation was set because we assume that the follow-up of bereaved in the health services after a suicide will mostly be directed towards the immediate family. Furthermore, the Treatpath project has two quantitative work packages based on longitudinal administrative and health registries linking each suicide in Norway to (immediate) family data. Hence, we have chosen to focus on bereaved in the immediate family.

\section{Stage 2: identifying relevant studies}

To identify relevant studies, we will use the populationconcept-context framework provided by the JBI for scoping reviews (table 1). ${ }^{19}$

This scoping review will include original research articles (quantitative, qualitative and mixed methods), as well as original articles identified via review articles, including systematic reviews, meta-analyses, meta-syntheses, narrative reviews, mixed-methods reviews and rapid reviews. Furthermore, we will use the CoCites citation-based search method on the systematic reviews identified. ${ }^{24}$ To be included, the studies must meet the following criteria:

\section{Table 1 Inclusion and exclusion criteria}

P- $\quad$ Suicide bereaved: immediate family (parents,

population spouse/partner, siblings and children of any age)

C- Different treatment trajectories in the health concept services and healthcare use such as primary care, specialised care, outpatient care, extramural care and formal support groups; only professional healthcare services will be included, and welfare services will not be included.

C-context Language limited to English, studies in developed countries defined as high-income countries according to the World Bank (2020), publication period in the past 10 years from January 2010 to June 2020.
(1) publication in a peer-reviewed journal, (2) original empirical study, (3) identification and inclusion of participants bereaved by suicide; (4) study aim included follow-up or health service use by families bereaved by suicide or health service use among the study outcomes, (5) publication between 2010 and 2020 and (6) study from a high-income country according to the World Bank.

We consider that the grey literature and use of welfare services by families bereaved by suicide are beyond the scope of this review, given the time frame and resources available in the Treatpath project. We will not include books or book chapters, reports or unpublished studies. We consider bereavement after euthanasia and physicianassisted suicide to be a different issue; therefore, we will not include studies focused primarily on this topic. Health services are constantly evolving, especially the past 10 years, with new technological tools and new ways of providing health services. As we wanted to capture 'up-to-date' follow-up in the health services, and development over time is not a central issue, we restrict the search to publications in the last 10 years. The focus on high-income countries was chosen to increase the probability of comparable levels of healthcare services in the included literature.

\section{Search strategy}

We established three key concepts to facilitate the search strategy: (1) suicide, (2) bereavement and (3) health services. In accordance with the three-step process suggested by the JBI, we first conducted a preliminary search in MEDLINE (via PubMed) and PsycINFO (via OVID) in May 2020, in which we limited the search to systematic reviews using the following search terms:

1. Suicide [Medical Subject Headings (MeSH)] AND Bereavement (MeSH).

\section{Suicid* AND Bereav*.}

Using this search strategy, we found 23 systematic reviews in PubMed (search conducted 18 May 2020) and 18 systematic reviews in PsycINFO (search conducted 18 May 2020). As examples, in 2018, Maple et al mapped the suicide bereavement and postvention literature from 1960 to $2017 .{ }^{16}$ In 2014, Andriessen et al examined suicide bereavement and postvention reported in the core international suicidology journals from 1971 to 2013. However, the focus in our scoping review will be patient pathways and follow-up of families bereaved by suicide, and we will include literature beyond 2017, which will be beneficial given the increasing number of articles on suicide bereavement in recent years. We used the systematic reviews from the preliminary search as inspiration when identifying our relevant search terms and developing our search strategy. We have consulted with a health sciences librarian at the Norwegian University of Science and Technology, Faculty of Medicine and Health Sciences, who helped us improve the search strategy.

For the full scoping review, we plan to conduct an extensive literature search using the following databases: MEDLINE (via Ovid), Embase (via Ovid), PsycINFO (via 
Table 2 Search strategy for concepts of (1) suicide, (2) bereavement and (3) health services

\section{Suicide Suicide $[\mathrm{MeSH}]$}

Suicid*

2.

Bereavement [Mesh]

3. Health services

bereave* OR grie* OR mourn*

Health Services [MeSH] OR Delivery of Healthcare [MeSH] OR Primary Healthcare [MeSH] OR Secondary Care [MeSH] OR Community Health Services [MeSH] OR Mental Healthcare [MeSH] OR Outpatient [MeSH] OR Inpatient [MeSH] OR Hospitals [MeSH] OR Ambulatory Care Facilities [MeSH] OR Ambulatory Care [MeSH] OR Counselling [MeSH] OR Referral and consultation [MeSH]

"healthcare" OR healthcare OR healthcare OR inpatient OR outpatient OR treat* OR support* OR follow-up OR "follow-up" OR counsel $^{\star}$ OR consult* OR hospital ${ }^{\star}$ OR ambulatory* OR team* OR postvention

MeSH, Medical Subject Headings.

Ovid), CINAHL (via EBSCO host) and Web of Science. We will use both subject headings (eg, MeSH, major heading, subject terms, Keywords Plus) and free-text searching. We will use a search strategy involving our three concepts as shown in table 2. An example of the search strategy in Ovid (APA PsycInfo, Embase and MEDLINE) is shown in the supplementary file.

\section{Stage 3: selecting the relevant studies}

After identifying the relevant studies in stage 2, articles and reviews will be exported from the databases and then imported into the EndNote X9 and Covidence applications. EndNote X9 is a reference-management software from Clarivate Analytics, while Covidence is available from the Cochrane Library. EndNote is used to remove duplicates and administrate references in forthcoming articles. We will use Covidence to to screen titles and abstracts efficiently. Two team members will participate in the process of screening titles and abstracts, according to our established eligibility criteria. The team comprises researchers with clinical experience and experience in health service research. The voting will be blinded (as is standard in Covidence), which means that the team members will not be aware of what the other members have voted with regard to eligibility. Where there is conflict, final decision on inclusion/exclusion will be made by discussion and consensus or by a third team member. Finally, we will also use the functions of Covidence to screen the full texts using the same method. As we work, the software will construct a Preferred Reporting Items for Systematic Reviews and Meta-Analyses (PRISMA) table automatically by keeping track of exclusions. We will include this PRISMA table in the final publication.

\section{Stage 4: charting the data}

We will chart the relevant studies in an evidence table, which will be updated continuously by all full-text readers. The skeleton of this chart will be provided by the export functions of Covidence.

For original research articles, we will include the following columns ('Data items'): author(s); year of publication; origin/country of origin (where the study was published or conducted); aims/purpose of the study; study population and sample size (if applicable); methodology/methods; outcomes and details of these (eg, how outcomes are measured) (if applicable); and key findings that relate to the scoping review question(s) (e.g. type, amount, timing and range of services/interventions, patient trajectories, patient experiences and outcomes). Data extraction will be done independently by four researchers.

To ensure that this framework will be appropriate and informative, we will pilot test it on a sample of the references comprising five original research articles from each database. If necessary, we will add or remove data items accordingly.

\section{Stage 5: collating, summarising and reporting the results}

We will use the checklist for reporting scoping reviewsthe PRISMA-ScR. ${ }^{25}$ We will use a thematic synthesis approach in this review, ${ }^{23}{ }^{26}$ given that the preliminary systematic review search we did showed that the literature on this research topic seems heterogenous in terms of the types of studies and choice of methods (ie, qualitative, quantitative and mixed methods). The thematic synthesis approach has three main steps: (1) coding the text of the studies, (2) developing descriptive themes and (3) generating the analytical themes. ${ }^{23}$ The reporting of results also involve providing key quantitative information, including country, publication year, study population, study type and health services involved. Reviewing the literature in this manner will enable us to identify important gaps in the general knowledge of families bereaved by suicide and may help to generate ideas for further research. This may prove to be especially useful for the rest of the Treatpath research project, which will conduct interviews with family members bereaved by suicide and registry-based observational studies on people who committed suicide and their immediate family members. This scoping review will not assess the quality of, or risk of bias in, the included studies.

\section{Stage 6: consultation}

Late in the process of stage 5, we will arrange a workshop/ team consultation with the Treatpath research team and The Norwegian Organisation for the Suicide Bereaved (involved throughout the project) to discuss the findings of the study and how to interpret and report them. This team comprises researchers, clinicians and user representatives (bereaved) with a variety of areas of expertise from different geographical locations in Norway. We will also discuss how to improve our methods. 


\section{ETHICS AND DISSEMINATION}

The methods of a scoping review aim only to scope and map previously published research, and ethical approval is generally not considered necessary. The review is part of a research project (Treatpath) that will include qualitative studies of the follow-up of families bereaved by suicide, as well as quantitative observational analyses of healthcare use in this group. The Treatpath project has been considered by the Regional Committee for Medical and Health Research Ethics (reference 2019/919) and has been ethically approved by the Norwegian Centre for Research Data (reference 924533). For dissemination, we aimed to publish our results in an international peer-reviewed scientific journal. We will share the knowledge gained from our findings at scientific conferences, in national journals and in social media. The National Association of Suicide Bereaved in Norway is systematically involved in the research project's advisory board and will help with the dissemination of the results to people bereaved by suicide, health services and health professionals.

Contributors Each author contributed as follows: SHP, JK and SLK conceived the study protocol and its design. SHP drafted the first version of the manuscript. JK and SLK provided input throughout the manuscript and revised the document according to comments from reviewers. All authors have contributed to and read the final manuscript and have agreed to its submission.

Funding The project is funded by the Research Council of Norway (grant number 288731).

Competing interests None declared.

Patient consent for publication Not required.

Provenance and peer review Not commissioned; externally peer reviewed.

Supplemental material This content has been supplied by the author(s). It has not been vetted by BMJ Publishing Group Limited (BMJ) and may not have been peer-reviewed. Any opinions or recommendations discussed are solely those of the author(s) and are not endorsed by BMJ. BMJ disclaims all liability and responsibility arising from any reliance placed on the content. Where the content includes any translated material, BMJ does not warrant the accuracy and reliability of the translations (including but not limited to local regulations, clinical guidelines, terminology, drug names and drug dosages), and is not responsible for any error and/or omissions arising from translation and adaptation or otherwise.

Open access This is an open access article distributed in accordance with the Creative Commons Attribution Non Commercial (CC BY-NC 4.0) license, which permits others to distribute, remix, adapt, build upon this work non-commercially, and license their derivative works on different terms, provided the original work is properly cited, appropriate credit is given, any changes made indicated, and the use is non-commercial. See: http://creativecommons.org/licenses/by-nc/4.0/.

\section{ORCID iD}

Sindre Hoff Petersen http://orcid.org/0000-0002-9066-3654

\section{REFERENCES}

1 Bachmann S. Epidemiology of suicide and the psychiatric perspective. Int J Environ Res Public Health 2018;15
2 World Health Organization. Mental health: suicide data, 2020. Available: https://www.who.int/mental_health/prevention/suicide/ suicideprevent/en/

3 Kawohl W, Nordt C. COVID-19, unemployment, and suicide. Lancet Psychiatry 2020;7:389-90.

4 Berman AL. Estimating the population of survivors of suicide: seeking an evidence base. Suicide Life Threat Behav 2011;41:110-6.

5 Cerel J, Brown MM, Maple M, et al. How many people are exposed to suicide? Not six. Suicide Life Threat Behav 2019;49:529-34.

6 Pitman A, Osborn D, King M, et al. Effects of suicide bereavement on mental health and suicide risk. Lancet Psychiatry 2014;1:86-94.

7 Andriessen K. Suicide bereavement and postvention in major suicidology journals: lessons learned for the future of postvention. Crisis 2014;35:338-48.

8 Andriessen K, Krysinska K, Grad OT. Postvention in action: the international handbook of suicide bereavement support. Postvention in action: the International Handbook of suicide bereavement support. Boston, MA: Hogrefe Publishing, 2017.

9 Linde K, Treml J, Steinig J, et al. Grief interventions for people bereaved by suicide: a systematic review. PLoS One 2017;12:e0179496.

10 Spillane A, Larkin C, Corcoran P, et al. Physical and psychosomatic health outcomes in people bereaved by suicide compared to people bereaved by other modes of death: a systematic review. BMC Public Health 2017;17:939.

11 Groot MHde, Keijser Jde, Neeleman J. Grief shortly after suicide and natural death: a comparative study among spouses and first-degree relatives. Suicide Life Threat Behav 2006;36:418-31.

12 Dyregrov K. Assistance from local authorities versus survivors' needs for support after suicide. Death Stud 2002;26:647-68.

13 Geulayov G, Gunnell D, Holmen TL, et al. The association of parental fatal and non-fatal suicidal behaviour with offspring suicidal behaviour and depression: a systematic review and meta-analysis. Psychol Med 2012;42:1567-80.

14 Kuramoto SJ, Runeson B, Stuart EA, et al. Time to hospitalization for suicide attempt by the timing of parental suicide during offspring early development. JAMA Psychiatry 2013;70:149-57.

15 Dyregrov K. How do the young suicide survivors wish to be Met by psychologists? A user study. Omega 2009;59:221-38.

16 Maple M, Pearce T, Sanford R, et al. A systematic mapping of suicide bereavement and postvention research and a proposed strategic research agenda. Crisis 2018;39:275-82.

17 Bartone PT, Bartone JV, Violanti JM, et al. Peer support services for bereaved survivors: a systematic review. Omega 2019;80:137-66.

18 Andriessen K, Krysinska K, Hill NTM, et al. Effectiveness of interventions for people bereaved through suicide: a systematic review of controlled studies of grief, psychosocial and suicide-related outcomes. BMC Psychiatry 2019;19:49.

19 Peters MDJ, Godfrey CM, Khalil H, et al. Guidance for conducting systematic scoping reviews. Int J Evid Based Healthc 2015;13:141-6.

20 Arksey H, O'Malley L. Scoping studies: towards a methodological framework. Int J Soc Res Methodol 2005;8:19-32.

21 Levac D, Colquhoun H, O'Brien KK. Scoping studies: advancing the methodology. Implement Sci 2010;5:69.

22 Moher D, Shamseer L, Clarke M, et al. Preferred reporting items for systematic review and meta-analysis protocols (PRISMA-P) 2015 statement. Syst Rev 2015;4:1.

23 Thomas J, Harden A. Methods for the thematic synthesis of qualitative research in systematic reviews. BMC Med Res Methodol 2008;8:45.

24 Janssens ACJW, Gwinn M, Brockman JE, et al. Novel citation-based search method for scientific literature: a validation study. BMC Med Res Methodol 2020;20:25.

25 Tricco AC, Lillie E, Zarin W, et al. A scoping review on the conduct and reporting of scoping reviews. BMC Med Res Methodol 2016;16:15.

26 Thomas J, Harden A, Oakley A, et al. Integrating qualitative research with trials in systematic reviews. BMJ 2004;328:1010-2. 